\title{
Studi sul Moyen Français A proposito degli Actes du IIIe Colloque International sur la Littérature en Moyen Français (Milan, 21-22 mai 2003)
}

\section{Anna Maria Finoli}

\section{(2) OpenEdition}

\section{Journals}

Edizione digitale

URL: http://journals.openedition.org/studifrancesi/8156

DOI: $10.4000 /$ studifrancesi.8156

ISSN: 2421-5856

\section{Editore}

Rosenberg \& Sellier

\section{Edizione cartacea}

Data di pubblicazione: 1 mai 2009

Paginazione: 91-101

ISSN: 0039-2944

\section{Notizia bibliografica digitale}

Anna Maria Finoli, «Studi sul Moyen Français A proposito degli Actes du Ille Colloque International sur la Littérature en Moyen Français (Milan, 21-22 mai 2003)», Studi Francesi [Online], 157 (LIII | I) | 2009, online dal 30 novembre 2015, consultato il 10 janvier 2021. URL: http://journals.openedition.org/ studifrancesi/8156 ; DOI: https://doi.org/10.4000/studifrancesi.8156

\section{(c) (i) (9)}

Studi Francesi è distribuita con Licenza Creative Commons Attribuzione - Non commerciale - Non opere derivate 4.0 Internazionale. 


\section{DISCUSSIONI E COMUNICAZIONI}

\section{Studi sul Moyen Français A proposito degli Actes du III Colloque International sur la Littérature en Moyen Français (Milan, 21-22 mai 2003)}

Gli studi sul periodo della lingua francese definito da Arsène Darmesteter e da Ferdinand Brunot moyen français, i cui termini cronologici vanno convenzionalmente dall'avvento al trono dei Valois (1328) a quello dei Borboni (1594), con qualche spostamento dei termini cronologici da parte di alcuni studiosi, hanno una storia relativamente recente, tanto che ancora nel 1988 si ritiene opportuno aprire i lavori del VI Colloquio Internazionale con una sintesi storica affidata a Marc Wilmet ${ }^{1}$. Come lo studioso belga ricorda, la ricerca sul moyen français inteso come momento autonomo della storia del francese risale agli anni Sessanta del Novecento. Fino ad allora opere fondamentali come i Vermischte Beiträge (1902-1912) di Adolf Tobler, i grandi lavori di insieme di Kristoffer Nyrop e di Ferdinand Brunot non dedicano particolare attenzione ai secoli XIV e XV. Nell'ambito lessicografico, dopo il Dictionnaire de l'ancienne langue française di Frédéric-Eugène Godefroy, che senza esitazioni annette al francese antico anche la lingua del Cinquecento, si crea poi uno iato tra l'Altfranzösisches Wörterbuch di Tobler-Lommatzsch e il Dictionnaire du XVI siècle di Edmond Huguet; analogamente, nell'ambito della sintassi si passa dalla Petite syntaxe de l'ancien français ( $1^{\mathrm{a}}$ ed., Paris 1919) di Lucien Foulet alla Grammaire de la langue française du XVI siècle (Lyon 1951) di Georges Gougenheim e alla Syntaxe française du XVII siècle (trad. franc., Paris 1898) di Alfons Haase. Dopo una prima, discussa, Brief Description of Middle French Syntax (Chapel Hill 1958) di Rosalyn Gardner e Marion A. Greene, uno dei primi lavori importanti in cui il moyen français appare come autonomo è La voix pronominale en ancien et en moyen français (Aix-en-Provence 1962) di Jean Stefanini, seguita poi da altri studi, particolarmente sul verbo, e, soprattutto, da un piccolo, intelligente libro di Pierre Guiraud, Le Moyen Français (Que sais-je?, Paris 1963), che offre una visione d'insieme e, in base a considerazioni strutturali, propone una tesi fortemente innovativa: il moyen français non sarebbe una fase dell'ancien français, ma un periodo di preparazione, una sorta di "berceau" in cui si fondano e si affermano le strutture della lingua moderna.

Come avviene quando si delineano nuovi campi di ricerca, gli studiosi interessati a queste tematiche si riuniscono per mettere in comune risultati e progetti. Dopo un primo incontro, una tavola rotonda, a Nancy (1974), patrocinato da Paul Imbs, si instaura la tradizione di colloqui a scansione triennale. A Düsseldorf (1980) si decide un'importante iniziativa: la pubblicazione di un Dictionnaire du Moyen Français, sotto la direzione di Robert Martin, concepito fin dall'inizio secondo i criteri della

(1) MARC WiLmet, Les études linguistiques: bilan et perspectives, in Actes du VIe Colloque Internatio- nal sur le Moyen Français, Milan 4-6 mai 1988, Milano, Vita e Pensiero 1991, vol. I, pp. 7-15. 
"lexicologie évolutive", cioè un dizionario su basi informatiche, di facile accesso e aperto a tutti gli apporti ritenuti opportuni ${ }^{2}$; una prima versione, infatti, è accessibile dal 2002 sul sito del Laboratorio ATILF (Analyse et Traitement Informatique de la Langue Française).

Dal 1977 esce, ad opera di Giuseppe di Stefano, una rivista, Le Moyen Français, dapprima a Palermo (nn. 1, 2, 4), dal 1980 a Montréal (nn. 3, 5-61), dal 2008 a Tournhout, dedicata a temi prevalentemente filologici e letterari, che dal 1984 pubblica gli atti dei congressi internazionali promossi presso la McGill University di Montréal con cadenza biennale; a essa si affianca una casa editrice specializzata (CERES) che pubblicherà, oltre ad una serie di testi inediti e rari, il prezioso Dictionnaire des Locutions en Moyen Français (1991) dello stesso Di Stefano e altri repertori lessicali. L'insieme di queste attività mostra come ormai anche la critica letteraria volga uno sguardo più aperto e più attento a questo momento della storia della cultura francese che, pur illustrato da personalità quali Villon e Charles d'Orléans, dal fiorire del teatro sacro e profano e dalla nascita della storiografia moderna, aveva per lungo tempo subito il pregiudizio sul Medio Evo, reso ancor più pesante dalla connotazione negativa della formula «autunno del Medio Evo». Anche tra gli storici ci si interroga sul carattere del periodo, travagliato da grandi turbamenti politici, economici, sociali, dalla guerra dei Cento Anni alle guerre di Religione, ma percorso anche da slanci vitali, da esigenze di rinnovamento del pensiero, dell'etica e dell'estetica, mentre si consolida, pur in alterne vicende, la monarchia centrale e si accresce l'importanza politica e culturale del ducato di Borgogna. E dunque, «Automne du Moyen Âge ou printemps?»: Michel Mollat giunge a indicare proprio nei secoli XIV e XV La génèse médiévale de la France moderne (Paris 1977).

Nel 1985, per la prima volta, il V Colloquio Internazionale, organizzato dal Centro Studi sulla Letteratura Medio-francese e Medio-inglese dell'Università Cattolica di Milano, riunisce filologi, linguisti e critici letterari'. La formula, ripresa nel 1988, comporta uno sforzo di comprensione tra metodi differenti che Jacques Monfrin, l'illustre e compianto medievista, non esiterà a definire «infiniment fécond» ${ }^{4}$.

Dal 1994 i due filoni di ricerca tornano a procedere separatamente: i linguisti tengono il loro VIII Congresso, Le moyen français. Philologie et linguistique. Approches du texte et du discours, a Nancy'; il IX, Le moyen français. Le traitement du texte (Edition, apparat critique, glossaire, traitement électronique), a Strasbourg, 19976; il X, Frédéric Godefroy, a Metz, 2002 ; 1'XI, Linguistique textuelle du moyen français, a Anvers/Antwerpen ${ }^{\text {. }}$.

Nel 2004 gli studiosi che gravitano intorno a Le Moyen Français, o che comunque ne condividono gli interessi, si costituiscono in associazione, l'Association Internationale pour l'Étude du Moyen Français, che ha lo scopo di facilitare gli scambi tra gli specialisti e incoraggiare la ricerca sulla letteratura e la lingua di questo periodo. L'AIEMF ha già organizzato tre convegni: il primo a Montréal (10-12 ottobre 2004) su Le langage figurê; ; il secondo a Poitiers (27-29 aprile 2006), su La traduction vers le moyen françai ${ }^{10}$; il terzo a Gargnano del Garda, sede dell'Università degli Studi di Milano (28-31 maggio 2008), su 'Mettre en prose' aux XIV'-XVI siècles. Approches linguistiques, philologiques, littéraires (Actes in corso di stampa, Tournhout, Brepols).

(2) R. Martin, Le Dictionnaire du Moyen Français, in «Cahiers de Lexicologie», 88, 2006-1, pp. 159-60.

(3) Actes, Milano, Vita e Pensiero 1986.

(4) Jacques Monfrin, Conclusion, in Actes, 1991, vol. III, p. 233.

(5) Actes, Paris, Didier Érudition, 1997.
(6) Actes, Presses Universitaires de Strasbourg 2000.

(7) Actes, Paris, École des Chartes, 2003.

(8) Actes, Tournhout, Brepols, 2007.

(9) Actes, in «Le Moyen Français», 60-61, 2007. 2007

10) Actes, in «The Medieval Translator», XI, 
Negli stessi anni presso l'Université Catholique de Louvain, culla degli studi, fondati da Georges Doutrepont, sulla cultura della corte di Borgogna, si forma un Groupe de recherche sur le moyen français, diretto da Claude Thiry, che promuove colloqui internazionali a scadenza biennale: il primo nel 2003, su La Littérature à la cour de Bourgogne. Actualités et perpectives de recherche $e^{11}$; il secondo nel 2005, su L'écrit et le manuscrit à la fin du Moyen Âge $e^{12}$; il terzo nel 2007 su Le recueil à la fin du Moyen Âge $e^{13}$.

Questi cenni di storia degli studi, che indicano alcune tappe importanti, quali la creazione degli strumenti fondamentali, l'elaborazione di uno statuto, l'estensione dell'interesse per la materia testimoniano un'acquisita autonomia, che permette di comprendere come si possa ormai affrontare senza giustificazioni teoriche temi generali come quelli di un'altra serie di Colloqui, sempre triennali, organizzati dalla Facoltà di Lingue e Letterature Straniere dell'Università Cattolica di Milano: ${ }^{\text {er }}, L^{\prime} H i$ storiographie (Milan, 5-7 mai 1997) ${ }^{14}$; $\mathrm{II}^{\mathrm{e}}$, La poésie lyrique (Milan, 8-10 mai 2000) $)^{15}$.

Gli Actes du III Colloque International sur la Littérature en Moyen Français (Milan, 21-22 mai 2003), La réception de la littérature en moyen français aux XVI ${ }^{e}$-XVII ${ }^{e}$-XVIII ${ }^{e}$ siècles, «in memoria di Gianni Mombello», a cura di Sergio Cigada, Anna Slerca, Giovanna Bellati, Monica Barsi, sono pubblicati, come i precedenti in «L'analisi linguistica e letteraria» ${ }^{16}$.

La formulazione del titolo del colloquio presenta qualche problema per la collocazione del XVI come primo nella sequenza dei secoli presi in considerazione, e quindi al di là dei termini cronologici convenzionali del moyen français. Il parametro adottato si giustifica da un punto di vista genericamente culturale, per il quale il Cinquecento è il secolo del Rinascimento e perciò un periodo a sé stante. Anche per quanto riguarda la storia della lingua, non mancano periodizzazioni che separano il secolo XVI dai due precedenti, e basti ricordare il periodico Le Français Préclassique (15001650) diretto da Jacques Abélard e soprattutto il lavoro fondamentale di Christiane Marchello-Nizia, Histoire de la langue française aux XIV et XV siècles (Paris 1979), la quale nell'Introduction della seconda edizione (Paris 1997), giustificando la sua scelta, traccia anche una sintesi delle diverse opinioni sull'argomento. Resta il fatto che la lingua del secolo è ancora, in gran parte, quella fluente dei secoli precedenti, anche se il mondo della cultura lato sensu (si pensi alla manualistica tecnica e alla tipografia) è percorso da tensioni alla normalizzazione grafica e morfologica e alla fissazione del lessico, che però si realizzeranno solo «au siècle de l'autorité (1594-1715)» ${ }^{17}$. Sarebbe stata dunque opportuna almeno qualche nota introduttiva agli Actes per illustrare la scelta dei confini cronologici della tematica del Colloquio.

Il volume si apre con due saggi di carattere generale: Le XVI siècle (pp. 9-35) di Sergio Cappello, e La réception de la littérature en moyen français au XVII siècle (pp. 37-76) di Thierry Delcourt, basati entrambi su una copiosa documentazione, di cui importa segnalare i risultati, anche se non si può renderne conto in tutti i particolari. L'autore del primo concentra la sua attenzione soprattutto sulla prima metà del secolo. La continuità della ricezione di opere dei due secoli precedenti è indicata dalla loro presenza nell'attività dei più importanti editori - tra i quali si distinguono i parigini Antoine Vérard, Galliot du Pré -, che da principio stampano prevalente-

(11) Actes, in «Le Moyen Français», 57-58, 20052006.

(12) Actes, Tournhout, Brepols 2006.

(13) Actes, Tournhout, Brepols 2008.

(14) Actes «à la mémoire de Jacques Monfrin», in «L'analisi linguistica e letteraria» VI, 1, 1998.
(15) Actes, in «L'analisi linguistica e letteraria» VIII, 1-2, 2000.

(16) Actes, in «L'analisi linguistica e letteraria» XII, 1-2, 2004.

(17) M. CoHEn, Histoire d'une langue: le français, Paris, Éditions sociales 1967, p. 175. 
mente opere storiche o edificanti, e autori del secolo xv, da Alain Chartier a Villon, ai Grands Rhétoriqueurs. Cappello si sofferma sui modi della trasmissione di queste opere da parte degli editori, che spesso intervengono sugli aspetti formali, soprattutto ammodernando la lingua: l'esempio più noto è la revisione delle edizioni di Villon, ultima quella ad opera di Marot. La lirica di ispirazione cortese costituisce un caso a parte: avendo come pubblico di riferimento la corte e l'aristocrazia, si trasmette ancora in forma manoscritta, circostanza che ne compromette la conservazione, come farà osservare G.M. Roccati ${ }^{18}$, ma appare anche in raccolte collettive, in cui si verifica una sostituzione progressiva della poesia del passato con quella di poeti più vicini nel tempo. Questa situazione propria del terzo e quarto decennio del secolo è analoga a quella delle edizioni dei romanzi in prosa: le versioni della grande tradizione cavalleresca negli anni Venti, la narrativa di origine spagnola a partire dagli anni Trenta, e infine, dopo la traduzione di Eliodoro da parte di Amyot (1548), i romanzi ispirati dal mondo greco, che rinnovano il panorama della letteratura francese. Sarebbe ingeneroso chiedere ad un contributo già così vasto e impegnato qualche riferimento ai mutamenti della situazione storica politica, culturale, religiosa in cui si verifica la ricezione delle opere considerate. Non si può che concordare sulle conclusioni di questo contributo, che indicano nel romanzo, eredità medievale filtrata da traduzioni, rifacimenti ed imitazioni, genere ancora «sans statut ni prestige théorique», un forte elemento di continuità.

L'autore del secondo saggio constata all'inizio che il secolo XVII è «celui de l'ignorance et de l'oubli», e tuttavia proprio la sua ricerca porta ad attenuare questo giudizio forse troppo drastico. Strumento di ricerca di Th. Delcourt sono i cataloghi degli editori, le fonti di informazione dei collezionisti privati, e gli inventari delle biblioteche private, in particolare di quella appartenuta ad una dinastia di membri del Parlamento di Digione. Nel primo gruppo prevalgono cronache e storia, che possono essere oggetto di edizioni "scientifiche" e di un interesse non tanto letterario, quanto "politico", che mira alla miglior conoscenza dei fasti nazionali, in particolare Commynes. Seguono nella graduatoria di frequenza la letteratura giuridica e quella cinegetica. Scarse sono le sopravvivenze letterarie e teatrali: tra i testi in prosa si trovano solo Les Quinze joyes de Mariage, Rabelais, e qualche edizione a basso prezzo di mises en prose di canzoni di gesta e di romanzi. La Bibliothèque bleue, costituita per un terzo da romanzi di cavalleria, è un caso a parte. Per quanto riguarda l'ambito privato, la letteratura medievale, raramente oggetto di stampa, è rappresentata da manoscritti, spesso miniati, i cui proprietari erano probabilmente più interessati all'oggetto prezioso in sé che al suo contenuto. L'ultimo paragrafo, in cui sono raccolte le reminiscenze di letture di opere dei secoli precedenti quali emergono negli scrittori, che sono anche lettori, completa il quadro della ricezione della letteratura tardo medievale nel XVII secolo: ancora una volta prevalgono i romanzi di cavalleria e gli storici.

Nathalie Dauvois ${ }^{19}$ segnala dapprima l'ambivalenza delle rappresentazioni emblematiche della Fortuna nelle arti figurative: da un lato la ruota, simbolo tipicamente medievale, che rimanda ad un'azione al di sopra dell'uomo; dall'altro varie immagini rinascimentali: uno scafo di cui un uomo tende la vela o una ninfa propizia che guida la nave tra onde tempestose; in esse l'uomo è invitato ad assumere un ruolo più attivo, a scegliere e a guidare la sua sorte. Le rappresentazioni letterarie sembrano seguire

(18) Cfr. saggio in questo volume di Actes, pp. 260-261.

(19) Nathalie Dauvois, Fortune entre Moyen
Âge et Renaissance, la roue, le labyrinthe et le chemin, ibid., pp. 77-94. 
uno schema analogo, anche se meno rigoroso. N.D. analizza poi secondo questa partizione le immagini della Fortuna nel Labyrinthe de Fortune di Jean Bouchet, con ampi riferimenti agli intertesti, per giungere alla definizione del testo come «mitoyen, fictif, versifié, intégrant et convertissant la tradition vernaculaire antérieure». Questo modo di riutilizzare immagini anteriori si ritroverà poi in due componimenti, un'elegia e una Complainte contre Fortune, dedicati entrambi al Cardinal de Châtillon, del Second Livre de Meslanges di Ronsard.

Un gruppo di comunicazioni concerne il romanzo in prosa La Belle Hélène de Constantinople. Barbara Ferrari ${ }^{21}$ si occupa della mise en prose anonima, trasmessa da tre manoscritti del secolo XV e da nove stampe del XVI, di cui una segnalata per prima dalla stessa Ferrari. Il numero delle edizioni, cui si può aggiungerne un'altra congetturata, e le informazioni fornite da carte d'archivio sono indicazioni di un successo che può apparire modesto rispetto a quello di altri testi dello stesso genere, successo dovuto soprattutto all'attenzione di un pubblico interessato in particolare alle componenti agiografica e patetica del racconto. Conclude il contributo l'analisi delle varianti, sia nell'organizzazione della materia, sia di quelle del testo delle stampe rispetto ai manoscritti, analisi che, condotta con grande acribia, testimonia a quali importanti conclusioni di ordine storico e culturale possa condurre questo strumento critico.

Altre due comunicazioni, Le programme iconographique de "La Belle Hélène de Constantinople" de Jehan Wauquelin. Rapport texte-image di Christine Bousquet-Labouérie (pp. 119-142) e Le programme iconographique de "La Belle Hélène de Constantinople" de Jeban Wauquelin di Marie-Claude de Crécy (pp. 143-178) sono di argomento simile, come denunciano i titoli. Inevitabilmente si sovrappongono, anche se diversamente modulati, alcuni argomenti, quali, ad esempio, la conformità delle immagini al testo, la preponderanza di elementi guerreschi e religiosi, il richiamo al contesto politico della Borgogna di Filippo il Buono, la tendenziale anglofilia, la presenza dello spirito di crociata. C.B.-L. si sofferma in particolare sulla descrizione di una battaglia navale, sulla rappresentazione delle città dalle caratteristiche architettoniche fiamminghe e sull'illustrazione di tre scene di battesimo. Nella conclusione l'autrice sottolinea la sensibilità dell'enlumineur Liédet, simile in questo agli altri miniaturisti fiamminghi, per i grandi avvenimenti contemporanei e la funzione non solo illustrativa, ma anche esplicativa delle immagini, che valorizzano il testo e conferiscono maggior profondità agli aspetti politici.

De Crécy presenta all'inizio l'analyse del romanzo, e le circostanze storico-culturali della redazione e dell'illustrazione, mettendo infine in luce i particolari rilevanti e aneddotici che spesso vanno oltre le indicazioni delle rubriche, ma si trovano nel corpo del testo. Con quest'opera Wauquelin e Liédet, ciascuno a suo modo e a suo tempo, hanno collaborato per fornire al duca Filippo un racconto "fondatore", come quelli che legittimano i regni di Francia e d'Inghilterra, che fornisca cioè antichità, e quindi dignità, ai possedimenti borgognoni del Nord. Completa il saggio una serie di luoghi tratti dall'edizione curata dalla stessa de $\mathrm{Crécy}^{21}$, in cui sono segnalate le corrispondenze con le immagini, e numerose riproduzioni di queste ultime.

G. Matteo Roccati ${ }^{22}$ redige un inventario ragionato e fino ad oggi esaustivo delle testimonianze, che comprendono anche testi inediti, della fortuna di Deschamps fino alle soglie del XIX secolo, che ragioni di spazio impediscono di citare qui. Questa

(20) Barbara Ferrari, La Belle Hélène de Constantinople au XVI siècle, ibid., pp. 95-117.

(21) Jehan Wauquelin, La Belle Hélène de Constantinople. Mise en prose d'une chanson de geste.
Éd. critique par Marie-Claude De Crécy, Genève, Droz, 2002, TLF 547.

(22) G. Matteo Roccati, Lectures d'Eustache Deschamps, in Actes du III Colloque, pp. 231-261. 
raccolta di dati permette all'autore alcune interessanti riflessioni conclusive. La fama e il prestigio di cui il poeta ha goduto presso i contemporanei si attenuano dopo la sua morte. La ricezione della sua immensa opera obbedisce ad una logica precisa e si svolge in tre tempi. Il primo (xv secolo e prima metà del XVI), in continuità con la trasmissione manoscritta originaria, vede la presenza di alcune ballate isolate, talvolta anonime, proposte per il loro contenuto diciamo così "morale" nei testi di altri autori. Il secondo (seconda metà del secolo XVI, XVII e parte del XVIII) si caratterizza per un lungo oblio, generato dalla mutata sensibilità estetica e dal pregiudizio negativo sul Medio Evo. Verso la fine del secolo XVIII un rinnovato interesse per la storia, anche dei secoli medievali, spinge gli eruditi alla riscoperta di Deschamps soprattutto come insostituibile fonte di conoscenza della vita del suo tempo (Sainte-Palaye). Ha così inizio un'attività di ricerca dapprima prevalentemente attenta agli aspetti storici, ma presto estesa a quelli letterari e filologici, che darà i suoi ultimi frutti, anche ecdotici, ai tempi nostri. La parabola della ricezione di Deschamps, comune ad altri autori, come Machaut, Froissart, e persino Charles d'Orléans, è determinata dal passaggio dal manoscritto alla stampa, la cui introduzione cristallizza la situazione culturale: si conserva e si trasmette la memoria di poeti, come Alain Chartier e Villon, che con la stampa appunto hanno acquisito lo statuto di autori, ma la stessa stampa, sostituendosi al manoscritto, porta all'eliminazione di ciò che è rimasto solo tale.

Alcuni contributi concernono la ricezione da parte degli eruditi dei secoli seguenti. Michel Stanesco ${ }^{23}$, tracciato in sintesi il quadro generale delle Recherches de la France, segnala l'innovazione di Pasquier, che definisce un'epoca non più secondo la successione regale, ma secondo una visione complessiva delle opere letterarie. Pasquier si colloca tra coloro che per patriottismo intendono "illustrare" la lingua materna, di cui riconoscono l'origine nella letteratura dei secoli medievali. Egli ricerca e scrive da storico, consapevole della mutevolezza delle cose umane, e da storico non assume l'atteggiamento di disprezzo verso il Medio Evo che era stato proprio del primo umanesimo.

Anne Schoysman ${ }^{24}$ segnala l'appartenenza di Fauchet all'importante corrente culturale che ritiene la storia letteraria e la storia della lingua, ma anche del diritto, parti di un'unità studiata e rappresentata per rivendicare l'indipendenza della Francia. La parte più interessante di questo contributo è costituita dall'analisi di un ms. di Fauchet (BnF, fr. 24726), in gran parte inedito, che raccoglie alcuni brevi e interessanti saggi critici, in particolare alcuni capitoli che non sono stati utilizzati nel Recueil de l'origine de la langue et de la poesie françoise, e che concernono autori dell'autunno del Medio Evo, quelli che, ad eccezione di Commynes, possono essere ricondotti al gruppo definito dalla critica moderna dei Grands Rhétoriqueurs. Non è però questo l'aspetto che interessa Fauchet, attento alle loro opere di storia, o affini, ciò che spiega la scelta, accanto ai Rbétoriqueurs, di Commynes, cui Fauchet, da storico, riconosce la qualità essenziale dello storico, l'obiettività.

Maria Colombo Timellii $i^{25}$ segnala l'interesse, di letterato, di narratore e di bibliofilo, del marchese di Paulmy per la letteratura del tardo Medio Evo, anche se il suo criterio di giudizio resta quello del suo secolo. Nella vasta raccolta di poeti e di prosatori, soprattutto di storici, offerta dai Mélanges tirés d'une grande bibliothèque, M.C.T. sceglie di esaminare, con attenzione filologica anche alla tradizione del testo,

(23) Michel Stanesco, Estienne Pasquier, lecteur des auteurs du XV' siècle, ibid., pp. 179-195.

(24) AnNe SCHOYSMAn, Le regard de Claude Fauchet sur le Moyen Age finissant, ibid., pp. 197-206.

(25) Maria Colombo Timelli, Le Marquis de
Paulmy lecteur et divulgateur des poèmes d'Olivier de La Marche: le "Chevalier deliberé" et le "Parement ou Triomphe des dames d'honneur", ibid., pp. 263-287. 
le pagine dedicate a Olivier de la Marche, nelle quali Paulmy traccia una breve bibliografia del poeta ed espone i contenuti dei due poemi del titolo. Dello Chevalier deliberé Paulmy sottolinea il carattere allegorico e il contenuto storico, apportando ulteriori notizie biografiche di alcuni personaggi citati. Al secondo poema, di cui dà un resoconto molto sintetico, Paulmy riconosce un prevalente interesse documentario sui cambiamenti della moda. Completa il saggio l'edizione di una breve notizia di La Curne de Sainte-Palaye sul Parement tratta dal ms. Moreau 1724 della BnF.

In Présence de la littérature en moyen français dans deux éditions de la "Bibliothèque Françoise" de La Croix du Maine (1584 et 1772), Pierre Demarolle ${ }^{26}$ confronta la prima edizione della Bibliothèque Françoise con quella del 1772, a cura di Rigoley de Juvigny, il quale non solo apporta aggiunte significative, ma soprattutto opera la fusione con la Bibliothèque Françoise di Antoine Du Verdier. Per meglio quantificare la presenza di autori del tardo Medio Evo, P.D. fa riferimento al classico Manuel bibliographique de la littérature française du Moyen Âge di R. Bossuat, traendone una serie di indicatori numerici (ad esempio, autori citati dal Bossuat e assenti nella prima edizione della Bibliothèque Française: 68 per cento, rapporto inverso: 42 per cento). Egli passa quindi ad esaminare i rapporti tra la letteratura e l'editoria, ciò che gli permette di concludere che la documentazione del bibliografo del Cinquecento è in grande misura fondata su opere a stampa.

Monica Barsi (Le Dictionnaire de Philibert Joseph Le Roux, un miroir du passé pour le XVIII siècle, pp. 305-322) individua in François Lacombe l'autore dell'edizione del 1786 del Dictionnaire di Leroux e lo colloca nel clima culturale del suo tempo. Lacombe, collaboratore di La Curne de Sainte-Palaye, rinnova l'opera di Le Roux, probabilmente d'accordo con l'editore del suo precedente Dictionnaire du vieux langage françois. M.B. analizza la nuova struttura del Dictionnaire, determinata dall'ampliamento di 102 voci e dall'aggiunta di 1282 lemmi, di 82 proverbi tratti dalla raccolta di Barbazan, di 25 proverbi di altra fonte, con interessanti riferimenti all'atteggiamento della cultura contemporanea verso il passato linguistico del paese, che può essere ricuperato anche per mezzo della ricezione lessicografica.

Due contributi concernono esempi particolari dei secoli XVI e XVII, quello di Gabriella Parussa, La réception aux XVI et XVII siècles d'un texte dramatique en moyen français: le "Mystère de la pacience de Job" ception du "Triparty en la science des nombres" (1484) de Nicolas Chuquet aux XVI et XVII siècles ${ }^{28}$.

G. Parussa rivolge la sua attenzione critica alle edizioni a stampa di un mystère della seconda metà del XV secolo, testimonianza del persistente interesse, al di là dei confini temporali convenzionali, verso opere teatrali di soggetto religioso. Per offrire un quadro dei processi di diffusione di un testo di questo genere, dedica a sei tra le otto edizioni conservate del mystère un rigoroso esame, che comprende anche la lingua e il contenuto di ciascuna e che tiene conto del fatto che le varianti delle edizioni potrebbero dipendere dal ricordo di qualche rappresentazione recente; ciò le dà anche la possibilità di proporre alcune correzioni del testo. Il caso della Pacience de Job conferma in parte alcune acquisizioni critiche, come lo spostamento dell'interesse del pubblico dalla rappresentazione alla lettura. G.P. illustra il percorso esemplare del-

(26) Pierre Demarolle, Présence de la littérature en moyen français dans deux éditions de la "Bibliothèque Françoise" de La Croix du Maine (1584 et 1772), ibid., pp. 289-303.

(27) Gabriella Parussa, La réception aux XVI et XVII siècles d'un texte dramatique en moyen français: le "Mystère de la pacience de Job", ibid., pp. $207-$ 229.

(28) Maria Teresa Zanola, La réception du "Triparty en la science des nombres" (1484) de Nicolas Chuquet aux XVI et XVII siècles, ibid., pp. 323-343. 
l'opera, dal teatro alla lettura individuale, dal pubblico delle città importanti a quello forse più popolare delle città più piccole e dei paesi di provincia.

Il saggio di Zanola affronta un aspetto particolare della ricezione, se non della letteratura, della cultura lato sensu che si è espressa in moyen français: la presenza di echi del trattato di Nicolas Chuquet nelle opere di Estienne de la Roche, Larismethique nouvellement composée (Lyon 1520 e 1538) e di Claude Gaspard Bachet de Méziriac, Problèmes plaisants et délectables qui se font par les nombres (Lyon 1612). Si tratta di un lavoro ricco di informazioni, anche in rapporto allo stato delle conoscenze di matematica e di matematica commerciale nei vari momenti storici, conoscenze di cui l'A., nella conclusione, non manca di sottolineare l'importanza, in una visione più ampia degli elementi economici e morali che attraverso il Medio Evo hanno laboriosamente foggiato l'uomo moderno.

La sezione riservata a Villon, consueta nei congressi dell'Università Cattolica dedicati al moyen français, si apre con un saggio di Anna Slerca, Villon et Pétrarque ${ }^{29}$. L'autrice riprende una questione trattata dalla critica solo raramente e mai sistematicamente, cioè l'influsso del Petrarca nell'opera di Villon, e concentra la sua attenzione sui possibili rapporti tra i Trionfi e quattro ballate, tre tratte dal Testament e una dai Poèmes variés. A.S. rileva qualche affinità strutturale e soprattutto tematica: la crudeltà dell'amata, l'ubi sunt, la vecchiezza e la morte. Mette poi a confronto le antitesi della ballata per il concorso di Blois e quelle utilizzate dal Petrarca nelle Rime; e infine istituisce un parallelo tra la Ballade pour prier Notre Dame e la Canzone alla Vergine. Si tratta di una ricerca di grande interesse, che non manca di porre degli interrogativi, data la diffusione già nel XV secolo della poetica petrarchesca, cui appartengono certi topoi e certi stilemi, e le molte zone d'ombra della biografia di Villon, che ci impediscono di ricostruire le sue letture personali: ci si chiede fino a che punto il suo "petrarcheggiare", per usare un'espressione di Giuseppe Di Stefano, possa essere il frutto di una conoscenza diretta dell'opera del poeta italiano o non piuttosto del petrarchismo imperante.

Il contributo di Rika Van Deyck, Les Testaments de Villon, une écriture ironi$q u e^{30}$, è costituito dall'analisi della tecnica dell'ironia nell'opera di Villon quale si legge nel ms. Coislin, di cui la stessa Van Deyck ha curato l'edizione informatica (Mallier, Saint-Aquilin-de-Pacy, 1974). In contrasto con T. Hunt, l'autrice riconosce nell'opera testamentaria villoniana non tanto una polifonia di voci che realizzerebbe l'ironia strutturale, quanto piuttosto la subordinazione di ciò che è eterogeneo ad un'argomentazione interamente omogenea; analizza quindi molto finemente tutte le modalità della scrittura ironica utilizzate da Villon: «l'ironie verbale (en énoncé)», su cui si sofferma con particolare attenzione e con ricchezza di citazioni, e «l'ironie situationelle ou du sort (hors énoncé)» a livello discorsivo; «l'ironie socratique»e el'ironie romantique» a livello metadiscorsivo.

Giovanna Bellati (Théodore de Banville et la ballade "à la manière de Villon") premette un esame della teoria sulla ballata esposta da Banville nel Petit Traité de poésie française, segnala quindi l'importanza che questa forma metrica occupa nella sua opera, e la presenza determinante del modello villoniano. Quanto al problema dei modi della trasposizione di tale genere, pur nel costante rispetto della struttura metrica in forme moderne, G.B. distingue e analizza quattro forme di trasposizione: il pastiche, utilizzato solo una volta nella Ballade des célébrités du temps jadis; la ripresa di un tema fondamentale, come nella Ballade des parisiennes, nella Ballade à sa

(29) Anna Slerca, Villon et Pétrarque, ibid., pp. 347-366.

(30) Rika Van Deyck, Les Testaments de Villon, une écriture ironique, ibid., pp. 367-399.

(31) Giovanna Bellati, Théodore de Banville et la ballade "à la manière de Villon", ibid., pp. 401-422. 
femme, Lorraine, che richiama la Ballade pour Robert d'Estouteville, e nella Ballade à la Sainte Vierge, in cui si fa espresso riferimento a Villon. Una terza forma consiste nel calco più o meno preciso di titoli villoniani, senza che il contenuto corrisponda; la quarta, costituita dal richiamo ad un motivo secondario, da un tratto stilistico o da una semplice citazione, è la più frequente. Sono, infine, messi in luce alcuni motivi non propriamente villoniani, ma solo genericamente medievali. Banville ha il merito di aver riproposto lo schema originario della ballata, in contrapposizione alla ballata romantica, e di aver creato un'immagine di Villon, emblema della tradizione nazionale, in contrasto con quella di poète maudit.

Chiude il volume un gruppo di comunicazioni su «sujets variés».

François Suard ("Aspremont" aux XV et XVI siècles) ${ }^{32}$ si propone di meglio definire la storia dello sviluppo della Chanson d'Aspremont a partire dal testo della fine del secolo XII, esaminando le opere francesi in prosa dal XIV secolo al XVI che sono giunte fino a noi. Sono prese in considerazione: la versione contenuta nelle Croniques et conquestes de Charlemaine di David Aubert (1458), le allusioni alla Geste nel Myreur des Histors di Jean d'Outremeuse (prima del 1400), una storia anonima di Gérard de Frattre che si legge nel ms. fr. 12791 della BnF (1530-1540). L'analisi comparativa, documentata e rigorosa, conduce F.S. a concludere che tutti gli autori dei rifacimenti hanno in comune la cura di collegare Aspremont ad altri testi, ciascuno attingendo a fonti diverse, adattate con diversi livelli di fedeltà; il più originale fra di essi è il rimaneggiatore del XVI secolo, che tenta di rinnovare i contenuti e soprattutto la scrittura della materia epica.

Jennifer Britnell (La Pragmatique Sanction dans des textes français du règne de Louis XII) ${ }^{33}$ premette che la Prammatica Sanzione (Bourges 1438), che, come è noto, definisce i diritti della Chiesa di Francia e i limiti del potere papale, pur costituendo un importante argomento della propaganda monarchica, non trova grande spazio nella produzione letteraria. J.B. esamina dapprima due testi, la Déploration de l'Eglise militante di Jean Bouchet e il Traicté de la différence des schismes et des conciles de l'Eglise di Jean Lemaire des Belges, apparsi negli anni 1510-1512, cioè in un periodo in cui era vivo il ricordo dell'immenso potere esercitato da Georges d'Amboise, legato pontificio e ministro del re. L'attività del cardinal d'Amboise in rapporto alla Prammatica Sanzione ha lasciato tracce anche in un gruppo di opere stampate a Parigi tra il 1507 e il 1509, tra le quali meritano in particolare di essere ricordati: una moralité, Le Nouveau Monde avec l'estrif du Pourveu et de l'Ellectif... (cfr. in questi stessi Atti il contributo di J. Koopmans), aspro attacco al ministro del re, e Les Abus du monde di Pierre Gringore, in cui le lodi del re si alternano alle riflessioni critiche sulla società.

Jelle Koopmans (Du nouveau sur "Le Nouveau Monde") ${ }^{34}$, dopo aver presentato i problemi critici d'ordine generale della moralité, procede ad un'analisi sistematica dei dati concernenti i testi riuniti dall'editore parigino sotto il titolo Le Nouveau Monde avec l'estrif du Pourvu et de l'Ellectif de l'Ordinaire et du Nommé; c'est ung livre bien renommé ensuivant la forme auctentique ordonnee par la Pragmatique. Si tratta di due opere, la Moralité (de l'Estrif) e la Sotise (du Nouveau Monde), testi che costituiscono un'acerrima difesa della Prammatica Sanzione contro la prepotenza papale e la debolezza regale. Elementi interni confrontati a dati storici confermano l'ipotesi, già proposta per la Sotise, di una rappresentazione di entrambe, in date diverse dello stesso anno (1508), a Tolosa, la cui università era animata dello stesso profondo spirito

(32) FranÇOIS SuARD, "Aspremont” aux XV et XVI" siècles, ibid., pp. 425-453.

(33) JenNifer BRITNELl, La Pragmatique Sanction dans des textes français du règne de Louis XII, ibid., pp. 455-469.

(34) Jelle Koopmans, Du nouveau sur "Le Nouveau Monde", ibid., pp. 613-631. 
gallicano delle due pièces. Quanto all'attribuzione, J.K. identifica l'autore in Jean, non André, de la Vigne, poeta e libraio tolosano.

Richard Trachsler (Des chiffres et des lettres. Observations sur l'orthographe de quelques scribes de la fin du Moyen Âge ${ }^{35}$ presenta in primo luogo i risultati di una ricerca di gruppo tendente a «comprendre ce que signifiait écrire et copier des textes littéraires à la fin du Moyen Âge». Dallo spoglio elettronico di testi i cui manoscritti sono attribuibili con una certa probabilità a copisti noti e attivi nel XV secolo è stato tratto un corpus di circa un milione di parole; il risultato del loro studio può essere compendiato in una serie di tre théorèmes che indico molto sommariamente: 1) i copisti hanno una sorta di «bon usage personnel»; 2) per definire il profilo ortografico di un copista contano le grafie costanti, ma anche quelle variabili; 3 ) l'exemplar da cui copia non influisce in maniera significativa sul sistema grafico del copista. La seconda parte del contributo è dedicata ad un esame della pratica scrittoria del copista Michel Gonnot, che si differenzia da altri per un atteggiamento più conservativo. Se non è possibile dar conto qui della minuziosa analisi delle grafie di questo copista, che opera in un momento tanto "fluido" del francese, è doveroso, invece, sottolineare la saggia prudenza con cui sono formulate le riflessioni conclusive sul carattere provvisorio dei risultati ottenuti.

Il saggio Quelques particularités syntaxiques de "Les Ditz des sages hommes" de Guillaume Tardif ${ }^{36}$, con il quale Gianni Mombello completa il suo intervento al Colloquio sul Moyen Français del $1985^{37}$, è uno degli ultimi scritti dell'insigne e compianto studioso. Si tratta di una rassegna sistematica delle particolarità sintattiche che offre l'opuscolo di Tardif, preceduta da qualche notizia biografica dello scrittore del Velay in funzione della lingua da lui usata. Lo spoglio accurato e l'analisi, poggiata su basi bibliografiche estese e sicure, condotta con un metodo critico che non esclude alcuna ipotesi - si vedano in particolare il paragrafo dedicato ai verbi (pp. 520-540) e la discussione sull'uso di par con le forme verbali in -ant (pp. 544-557) - ne fanno un lavoro prezioso per la conoscenza della lingua di un autore come Tardif, testimonio della cultura francese nella seconda metà del secolo XV. Il saggio costituisce anche uno strumento esemplare, un termine ineludibile di raffronto per lo studio della lingua di altri autori contemporanei, e quindi per una conoscenza più completa del moyen français, questo momento così complesso e sfuggente della storia del francese. Proprio la lucida consapevolezza del problema detta a Mombello le considerazioni conclusive con l'invito agli editori critici ad usare grande prudenza nell'intervenire sui testi, virtù sempre auspicabile, ma assolutamente indispensabile nei confronti di una lingua che non ha ancora trovato regole sicure e accettate dalla comunità culturale.

Patrizio Tucci (Une poésie sépulcrale au $X V^{e}$ siècle) ${ }^{38}$ traccia dapprima un regesto delle opere o parti di opere che possono costituire un filone particolare della letteratura del secolo Xv: Le chevalier deliberé di Olivier de la Marche, Le Temple de Bonne Renommee di Jean Bouchet, Le Livre du Cuer d'Amours espris di René d'Anjou, Le Champion des Dames di Martin Le Franc, l'Hospital d'Amour di Achille Caulier, Le Temple de Boccace, L'oultré d'Amour e La complainte d'Hector di Georges Chastelain. L'A. sottolinea che, non distinguendosi tali testi per un'unica struttura formale, criterio fondamentale di identificazione e differenziazione è il contenuto, in cui si intrec-

(35) Richard Trachsler, Des chiffres et des lettres. Observations sur l'orthographe de quelques scribes de la fin du Moyen Âge, ibid., pp. 471-490.

(36) Gianni Mombello, Quelques particularités syntaxiques de "Les Ditz des sages hommes" de Guillaume Tardif, ibid., pp. 491-560.
(37) Gianni Mombello, «Les ditz des sages hommes de Guillaume Tardif». Aspects littéraires et linguistiques, in Actes 1986, pp. 199-216.

(38) Patrizio Tucci, Une poésie sépulcrale au XV siècle, in Actes du IIIe Colloque, pp. 561-592. 
ciano inestricabilmente tre dimensioni: la descrittiva, l'ideologica e l'elegiaca. L'esame dei testi indicati è ripreso e approfondito in due lunghe sezioni, Morphologies e Des lieux de mémoire, e in un più breve paragrafo, «Un si précieux cimetiere»; gli aspetti retorici, in particolare l'ekphrasis, e i legami con la cultura, l'ideologia e la mentalità del tempo sono illustrati con una fitta rete di dotti riferimenti alla cultura contemporanea utilizzati con grande sicurezza metodologica.

Nella comunicazione Les rapports de force dans la liaison amoureuse chez Christine de Pizan, Alain Chartier et Antoine de La Sale ${ }^{39}$, Rosalind Brown-Grant muove dalle recenti posizioni critiche nel dibattito sui rapporti di forza tra i due sessi nell'amore cortese. Dopo aver ricordato l'importanza del Roman de la Rose di Jean de Meun e del De amore di Andrea Cappellano per quanto riguarda la rappresentazione di tale rapporto nel tardo Medio Evo, offre una lettura particolarmente attenta agli aspetti della retorica cortese, specialmente alla tematica dello sguardo, definito "narcisistico", elemento fondamentale dell'innamoramento, del Livre du Duc des vrais amans di Christine de Pizan, della Belle Dame sans Merci di Alain Chartier e del Petit Jean de Saintré di Antoine de La Sale. R. B.-G. mette poi a confronto le diverse interpretazioni dei tre autori, tanto dei problemi dell'incompatibilità tra l'onore maschile e quello femminile quanto del rapporto di forza nell'amore cortese. Per Christine anche un personaggio positivo come il duca può distruggere la vita di una donna, che, pur gelosa del suo onore, si perde consentendo ai desideri dell'amante; secondo Chartier in amore sono gli uomini le vittime della durezza di cuore delle donne, ma anche "la belle Dame sans merci" è in fondo oggetto di biasimo per la sua scelta crudele; in La Sale il rapporto cortese tra la domna e il vassallo rischia di degenerare in una lotta violenta, e la protagonista subisce un destino funesto.

Per la ricchezza e la varietà dei contributi, questi Atti costituiscono una rinnovata testimonianza dell'interesse degli studi nell' ambito della letteratura e in generale della cultura che si è espressa in moyen français; sono così prezioso strumento di lavoro e insieme invito ad ulteriori ricerche e approfondimenti.

ANNA MARIA FINOLI

(39) Rosalind Brown-Grant, Les rapports de force dans la liaison amoureuse chez Christine de
Pizan, Alain Chartier et Antoine de La Sale, ibid., pp. 593-611. 